\title{
Constraints of the entropic index of Tsallis statistics in the context of hadronic collisions at ultra high energies using measures of the depth of maximum of air showers
}

\author{
M. Abrahão ${ }^{a}$, W. G. Dantas ${ }^{a}$, R. M. de Almeida ${ }^{a *}$, J. C. dos Anjos ${ }^{b}$ \\ ${ }^{a}$ EEIMVR - Universidade Federal Fluminense \\ ${ }^{b}$ Centro Brasileiro de Pesquisas Físicas \\ E-mail: rmenezes@id.uff.br
}

\begin{abstract}
Cosmic rays of ultra-high energy are one of the great puzzles of modern physics. So far their production mechanisms, sources, chemical composition and abundances as a function of energy are unknown. One can infer the primary mass composition from the depth of maximum, $X_{\max }$, of the longitudinal development of air showers induced by cosmic rays. Measurements of the evolution of this observable as a function of energy performed by the Pierre Auger Collaboration indicate the presence of a heavier component in the absence of new physics in the range of 100 $\mathrm{TeV}$ at the center of mass frame. These results are sensitive to the dynamics of the first interaction and depend on the validity of the hadronic interaction models extrapolated from data at lower energies. Additionally, recent data from the Large Hadron Collider (LHC) at CERN, for $p-p$ and A-A collisions at center of mass energy $\sim \mathrm{TeV}$ indicate a significant increase on the multiplicity of hadrons produced with respect to the multiplicity predicted by models generally used. This result, as well as the transverse momentum distribution of the particles produced in the collision are parameterized by the Tsallis distribution favoring a thermodynamic/statistical interpretation of the hadronization process. In this work we constrain the values of the entropic index $q$ of the Tsallis model by comparing its $X_{\max }$ predictions with measurements performed by the Pierre Auger Collaboration, under the assumption of a proton primary composition.
\end{abstract}

The 34th International Cosmic Ray Conference,

30 July- 6 August, 2015

The Hague, The Netherlands

\footnotetext{
* Speaker.
} 


\section{Introduction}

The Pierre Auger Observatory have led to great discoveries in the field of ultra-high energy cosmic rays (UHECRs) such as the confirmation of a suppression of the cosmic ray flux at energies above $4 \times 10^{19}[1,2,3] \mathrm{eV}$, limits on photon $[4,5,6,7]$ and neutrino fluxes $[8,9,10,11]$ at ultrahigh energies and a hint of large scale anisotropies at energies above $8 \mathrm{EeV}$ [12]. Nevertheless many questions related to these particles are still open. Particularly interesting is the observation of the unexpected behavior of the depth of shower maximum with energy. Interpreted with the leading LHC-tuned shower models, this implies a gradual shift to a heavier composition, with large fraction of protons at $10^{18} \mathrm{eV}$, changing to a heavier composition at $10^{19.5} \mathrm{eV}$ [13]. However, we should interpret this result with caution since measurements of shower properties performed by Auger Collaboration have revealed inconsistencies among data and present shower models and so this unexpected behavior of the depth of shower maximum evolution could be originated by new hadronic interaction physics at energy scales beyond the reach of LHC.

Recently a power-law function based on the Tsallis statistics [14] has been widely used in fits of the transverse momemtum $\left(p_{T}\right)$ and pseudo-rapidity $(\eta)$ distributions measured in high-energy collisions $[15,16,17,18,19]$ while several studies have been devoted to discuss these results in the literature [20, 21, 22, 23, 24, 25, 26, 27, 28]. The Tsallis statistics, extensively used in different branches of science, are used whenever a system presents memory effects, experiences long range correlations, intrinsic fluctuations, has fractal structures or its phase space is limited. It consists in replacing the classical Boltzmann-Gibbs entropy $\left(S_{B G}\right)$ by the form proposed by Tsallis

$$
S_{q}=\frac{\left(1-\sum_{i} p_{i}^{q}\right)}{q-1} \stackrel{\mathrm{q} \rightarrow 1}{\Longrightarrow} S_{B G}=-\sum_{i} p_{i} \ln p_{i} .
$$

Therefore, the entropy $S_{q}$ is nonextensive because

$$
S_{q}(A+B)=S_{q}(A)+S_{q}(B)+(1-q) S_{q}(A) S_{q}(B),
$$

with the parameter $q$ being a measure of the nonextensivity of the system. As a consequence, we must replace the usual Boltzmann exponential by the Tsallis power law distribution

$$
\exp \left(-\frac{E}{T}\right) \Longrightarrow \exp _{q}\left(-\frac{X}{T}\right)=\left[1-(1-q) \frac{E}{T}\right]^{\frac{1}{1-q}} .
$$

Following the ideas presented in [28], we suppose in this work that the reason for the introduction of Tsallis statistics in the context of ultra high energy collistion is the presence of statistical fluctuations intrinsic to the system. Thus, it can be shown that fluctuations of the parameter $T^{-1}$ according a gamma function $\Gamma(x)$ lead in a natural way to the $q$-exponential Tsallis power law distribution given in equation (1.3) with

$$
q=1+\frac{\sigma_{T}^{2}}{\langle T\rangle^{2}}=1+\frac{\left\langle\left(\frac{1}{T}\right)^{2}\right\rangle-\left\langle\left(\frac{1}{T}\right)\right\rangle^{2}}{\left\langle\left(\frac{1}{T}\right)\right\rangle^{2}} .
$$

Notice that in the absence of fluctuations of the temperature, $q=1$, and we return to the BolzmannGibbs equilibrium at temperature $T$. 
The transverse momentum and pseudo-rapidity distributions resulting from high energy collisions measured by several experiments show a large discrepancy in the values of the parameter $T$, reflecting different physics for the transverse and the longitudinal space. The transverse distributions are themal-like, presenting a parameter $T_{T}$ almost independent of the energy while those from the longitudinal space have a temperature $T_{L}$ sensitive to the energy of the collision, understood as the mean energy available per produced particle [28]. As discussed in that paper, since the data obtained from high energy particle physics experiments are averaged over many events, two scenarios related to the intrinsic fluctuations are possible:

(a) The temperature $T$ is constant in each collision but it fluctuates event by event: in this case the distribution of the particles generated in the high energy interaction has an exponential depedence (Boltzmann-Gibb distribution) since the system reached equilibrium. In this scenario only the resulting energy distribution from many collisions follows a Tsallis distribution. Indeed this is observed by particle physicists in accelerators.

(b) The temperature $T$ fluctuates within each collision: in this case we expect that the energy distribution of the particles generated in a single high energy interaction follows a power law Tsallis distribution, as discussed before.

Moreover, since the measured Tsallis index for the longitudinal space $q_{L}$ is much larger than measured for the transverse space $q_{T}$, the resulting $q \sim q_{L}$ and therefore it can be shown that both cases ((a) and (b)) lead to a Negative Binomial multiplicity distribution given by

$$
P(N)=\frac{(q-1)^{N}}{N !} \cdot \frac{q-1}{2-q} \cdot \frac{\Gamma\left(N+1+\frac{2-q}{q-1}\right)}{\Gamma\left(\frac{2-q}{q-1}\right)} \cdot\left(\frac{E}{T_{L}}\right)^{N}\left[1-(1-q) \frac{E}{T_{L}}\right]^{-N+\frac{1}{1-q}} .
$$

The parameter $q=q_{L}$ is the measure of fluctuations over $T_{L}=\frac{k \sqrt{s}}{\langle N\rangle}$, where $\langle N\rangle, k$ and $\sqrt{s}$ are, respectivelly, the mean multiplicity, the inelasticity and the total energy of the collision in the center of momentum frame.

In this work we consider only the scenario (a). The study of scenario (b) is in progress and will be adressed elsewhere. Here we present simulation results for the slant depth of the maximum $\left(X_{\max }\right)^{1}$ of air showers initiated by the collision of a proton primary particle with nucleus of the upper atmosphere, aiming to constrain the Tsallis index $q_{L}$ by comparing the results of the simulations with measurements reported by the Pierre Auger Collaboration [13].

\section{Simulations}

For all simulations presented in this work, we have used CORSIKA 6.981 [29] with the interaction models Sibyll 2.1 [30] and GHEISHA 2002d [31], for high and low energy processes, respectively. The air shower simulation chain is as follows: first we simulate the secondaries generated in the collision between a proton and a nucleus of the upper atmosphere externally by

\footnotetext{
${ }^{1}$ The slant depth of the maximum in the atmosphere is defined as the integral of the atmospheric density along the shower axis $\int \rho d x$ and expressed in units of $\mathrm{g} / \mathrm{cm}^{2}$ at which the shower reaches its maximum development, $X_{\max }$. For brevity, from now on we will refer to the slant depth as simply depth. However, the reader should be aware that in the literature the term depth may be used to refer to the integral of the atmospheric density along the vertical and not along the shower axis.
} 
assuming that the hadronization process is described by statistical equilibrium added to fluctuations of mean multiplicity; the resulting particle list is then inserted back into CORSIKA (using the stacking option) to proceed with usual cascade development through the atmosphere. We have used CORSIKA sampling option with thinning $=10^{-6}$. Given the lack of knowledge of the mean multiplicity at ultra high energies, such a procedure was performed 200 times for several values of $q_{L}(1.01,1.05,1.10,1.50$ and 1.90$)$ and $\langle N\rangle(240,300,360$ and 420 particles) with a primary proton of fixed energy $E=10^{18} \mathrm{eV}$ and zenith angle $\theta=38^{\circ}$. This model means that for lower energies, the Sibbyl predictions are good, since they are tuned by accelerator data, while it fails for higher energies $\left(\gtrsim 10^{18} \mathrm{eV}\right.$ ), and so, we are forced to use the Tsallis statistics.

\subsection{Simulations of first secondaries}

The point of the first interaction between the proton primary particle and a nucleus of the upper atmosphere is determined using the $p$-Air cross-section predicted by the Sibyll model and, as the $T_{L}$ is related with the fluctuations of mean multiplicity, we use, for simplicity, inelasticity $k=1$ $\left(T_{L}=\sqrt{s} /\langle N\rangle\right)$. Since we verified through simulations that the $\left\langle X_{\max }\right\rangle$ is not sensitive to $q_{T}$, all simulations presented in this work were evaluated with $q_{T}=1.01$ and $\left\langle T_{T}\right\rangle=133 \mathrm{MeV}$. Therefore, the parameter that we want to constrain from this analysis is $q_{L}$, related to the fluctuations of $T_{L}$, or, in other words, of the mean multiplicity $\langle N\rangle$. For each pair of $q_{L}$ and $T_{L}(\langle N\rangle)$, we first smear the temperature $T_{L}=\left\langle T_{L}\right\rangle$ by a gamma function

$$
f\left(\frac{1}{T_{L}}\right)=\frac{1}{\Gamma\left(\frac{1}{q-1}\right)}\left(\frac{T_{L}}{q-1}\right)\left(\frac{\left\langle T_{L}\right\rangle}{(q-1) T_{L}}\right)^{-\frac{q}{q-1}} \exp \left(\frac{-\left\langle T_{L}\right\rangle}{(q-1) T_{L}}\right) .
$$

Therefore, the resulting $T_{L}$ is the temperature of equilibrium of the fireball formed in the $p$-Air high energy collision. Then, we generate $N$ independent particles with energies $\left\{E_{1, \ldots}, E_{N}\right\}$ each one distributed according to the Boltzmann distribution

$$
f\left(E_{i}\right)=\frac{1}{T_{L}} \exp \left(-\frac{E}{T_{L}}\right)
$$

with the constraint $E=\sum_{i=1}^{N} E_{i}$. We proceed in the same way with respect to the tranverse space $\left(q_{T}\right.$ and $\left.T_{T}\right)$. When the interaction reaches equilibrium, the transverse momentum distribution for each collision is obtained using the Hagedorn Model [32]

$$
\frac{d N}{d p_{T}} \simeq c p_{T} \exp \left(-\frac{p_{T}}{T_{T}}\right)
$$

The type of particles are randomly generated according to Sibyll predictions and once we have generated the particles masses $m$, the longitudinal momentum is obtained as $p_{L}=\sqrt{E^{2}-m^{2}-p_{T}^{2}}$. These kinematical variables complete all the information we need to reintroduce in CORSIKA to proceed with shower propagation through the atmosphere.

\section{Results}

We show in figure 1 the $X_{\max }$ distributions obtained from air shower simulations initiated by interaction between a proton of $E=10^{18} \mathrm{eV}$ and a nucleus of the upper atmosphere in which 
the hadronization process is described by statistical equilibrium added to fluctuations of mean multiplicity for different $q_{L}$ values and mean multiplicities: $\langle N\rangle=240$ (top left), $\langle N\rangle=300$ (top right), $\langle N\rangle=360$ (bottom left) and $\langle N\rangle=420$ (bottom left). Summarizing the results, figures 2 and 3 present the mean $\left\langle X_{\max }\right\rangle$ and $\operatorname{RMS}\left(X_{\max }\right)$ obtained from those distributions. Although these results have been obtained for a fixed primary energy of $E=10^{18} \mathrm{eV}$, we can compare them, at least roughly, with those reported by the Auger Collaboration for two energy bins: $\left\langle X_{\max }\right\rangle=$ $719.9 \pm 1.4_{-10.2}^{+7.5}$ and $\operatorname{RMS}\left(X_{\max }\right)=62.4 \pm 2.1_{-1.8}^{+2.1}$, for the $\left[10^{17.9} \mathrm{eV}, 10^{18.0} \mathrm{eV}\right)$ energy range, and $\left\langle X_{\max }\right\rangle=725.2 \pm 1.5_{-10.2}^{+7.4}$ and $\operatorname{RMS}\left(X_{\max }\right)=59.5 \pm 2.0_{-1.9}^{+2.2}$, for the $\left[10^{18.0} \mathrm{eV}, 10^{18.1} \mathrm{eV}\right)$ energy range. Simple combinations of both bins using the weighted average are represented by the solid red lines in figures 2 and 3 with a rough estimation of the $3 \sigma$ statistical uncertainty band around them. Considering these plots, one can see that there is no value of $q_{L}$ compatible with the $\left\langle X_{\max }\right\rangle$ and $\operatorname{RMS}\left(X_{\max }\right)$ data at the same time for $\langle N\rangle=240$ and $\langle N\rangle=420$. On the other hand, all $q_{L}$ except $q_{L}=1.1$ are discarded for $\langle N\rangle=300$. Besides, the results for the mean multiplicity $\langle N\rangle=360$ present more $q_{L}$ values consistent with data, although the mean values for $q_{L}=1.01$, 1.50 and 1.90 are almost out of the band. Indeed $\langle N\rangle=360$ is the mean multiplicity predicted by the Sibyll model for a primary proton with $E=10^{18} \mathrm{eV}$. Furthermore, if we take into account the systematic uncertainties, $\sigma_{\text {stat },\left\langle X_{\max }\right\rangle} \sim 10 \mathrm{~g} / \mathrm{cm}^{2}$ and $\sigma_{\text {stat }, R M S\left(X_{\max }\right)} \sim 2 \mathrm{~g} / \mathrm{cm}^{2}$, the constraint on $q_{L}$ values is weakened with no $q_{L}$ being rejected from $\left\langle X_{\max }\right\rangle$ distributions and only few of them from the $\operatorname{RMS}\left(X_{\max }\right)$ analysis.

\section{Conclusions}

Although the simulations presented in this work are a very simple description of ultra high energy interactions, the preliminary results presented here show that the mean slant depth of the maximum $\left\langle X_{\max }\right\rangle$ and its fluctuation $\operatorname{RMS}\left(X_{\max }\right)$ are sensitive to intrinsic fluctuations of the system with respect of mean multiplicity $\langle N\rangle$ given by the parameter $q_{L}$. If the mean interaction multiplicty fluctuates due to some different initial conditions, it favors a $q_{L}$ value $\sim 1.1$ in case of $\langle N\rangle=300$ while it does not reject $q_{L}=1.01,1.50$ and 1.90 for $\langle N\rangle=360$. All values of $q_{L}$ are rejected for $\langle N\rangle=240$ and $\langle N\rangle=420$. If we take into account the systematic uncertainties, $\sigma_{\text {stat },\left\langle X_{\max }\right\rangle} \sim 10$ $\mathrm{g} / \mathrm{cm}^{2}$ and $\sigma_{\text {stat }, R M S\left(X_{\max }\right)} \sim 2 \mathrm{~g} / \mathrm{cm}^{2}$, the constraint on $q_{L}$ values is weakened with no $q_{L}$ being rejected from $\left\langle X_{\max }\right\rangle$ distributions and only few of them from the $\operatorname{RMS}\left(X_{\max }\right)$ analysis. Future studies regarding scenarios in which the temperature fluctuates within collision are in progress and will be reported in a future work. 

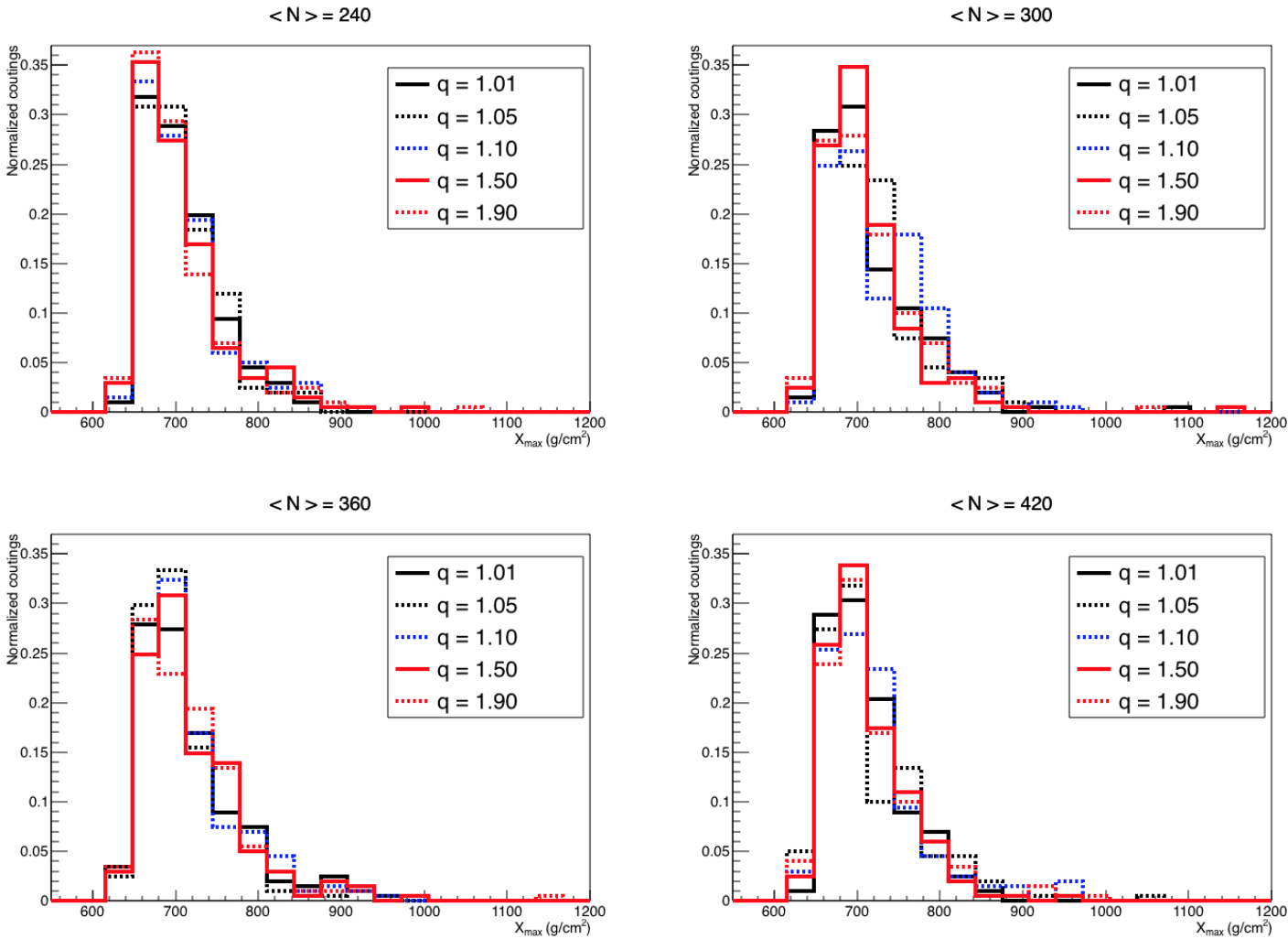

Figure 1: $X_{\max }$ distributions obtained from air shower simulations initiated by the interaction between a proton of $E=10^{18} \mathrm{eV}$ and a nucleus of the upper atmosphere in which the hadronization process is described by statistical equilibrium added to fluctuations of mean multiplicity for different $q_{L}$ values and mean multiplicities: $\langle N\rangle=240$ (top left), $\langle N\rangle=300$ (top right), $\langle N\rangle=360$ (bottom left) and $\langle N\rangle=420$ (bottom right).

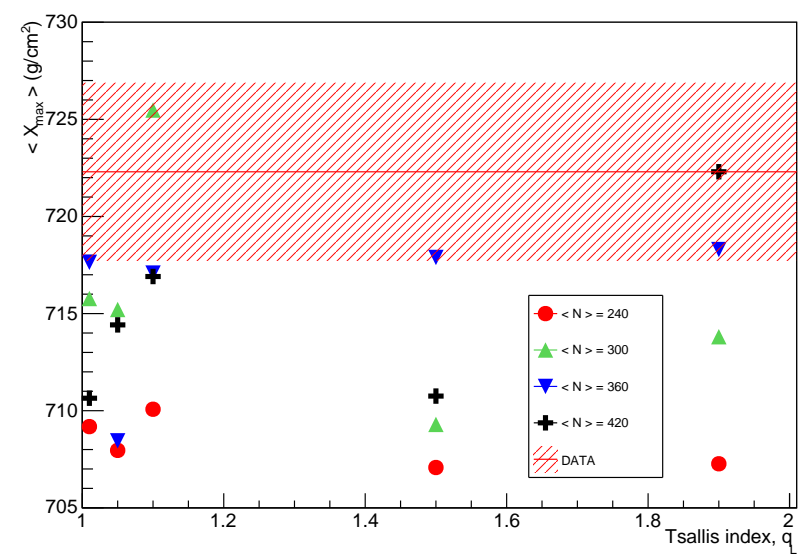

Figure 2: Expected mean $X_{\max }$ values from showers initiated by the interaction between a proton of $E=10^{18}$ $\mathrm{eV}$ and a nucleus of the upper atmosphere in which the hadronization process is described by statistical equilibrium added to fluctuations of mean multiplicity. The horizontal solid line represents the result measured by the Pierre Auger Collaboration with a rough estimation of its $3 \sigma$ band. 


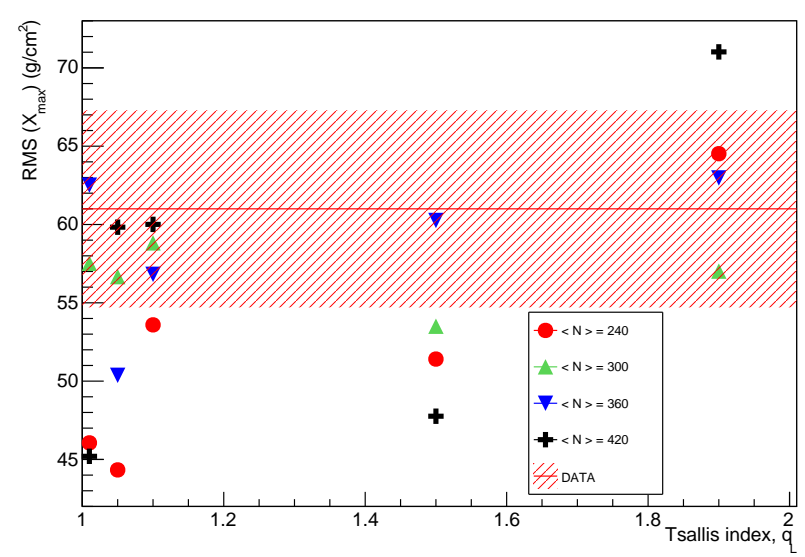

Figure 3: Expected $\operatorname{RMS}\left(X_{\max }\right)$ values from showers initiated by the interaction between a proton of $E=$ $10^{18} \mathrm{eV}$ and a nucleus of the upper atmosphere in which the hadronization process is described by the non extensive Tsallis statistic. The horizontal solid line represents the result measured by the Pierre Auger Collaboration with a rough estimation of its $3 \sigma$ band.

\section{Acknowledgments}

This work was partially supported by CNPq and FAPERJ.

\section{References}

[1] Pierre Auger Collaboration, J. Abraham et al.,"Observation of the suppression of the 4395 flux of cosmic rays above $4 \times 10^{19} \mathrm{eV}^{\prime \prime}$, Phys. Rev. Lett. 101 (2008) 061101, arXiv:0806.4302 [astro-ph].

[2] Pierre Auger Collaboration, J. Abraham et al., "Measurement of the energy spectrum of cosmic rays above $10^{18}$ eV using the Pierre Auger Observatory", Phys. Lett. B 685 (2010) 239-246, arXiv:1002.1975 [astro-ph.HE].

[3] Pierre Auger Collaboration, A. Aab et al., "Measurement of the cosmic ray spectrum above $4 \times 10^{18}$ eV using inclined events detected with the Pierre Auger Observatory", arXiv:1503.07786 [astro-ph.HE].

[4] Pierre Auger Collaboration, J. Abraham et al.,"An upper limit to the photon fraction in cosmic rays above $10^{19}$ eV from the Pierre Auger observatory", Astropart. Phys. 274405 (2007) 155-168, astro-ph/0606619.

[5] Pierre Auger Collaboration, J. Abraham et al.,"Upper limit on the cosmic-ray photon flux above $10^{19}$ eV using the surface detector of the Pierre Auger Observatory", Astropart. Phys. 29 (2008) 243-256, arXiv:0712.1147 [astro-ph].

[6] Pierre Auger Collaboration, J. Abraham et al.,"Upper limit on the cosmic-ray photon fraction at EeV energies from the Pierre Auger Observatory", Astropart. Phys. 31 (2009) 399-406, arXiv:0903.1127 [astro-ph.HE].

[7] Pierre Auger Collaboration, M. Settimo et al.,"An update on a search for ultra-high energy photons using the Pierre Auger Observatory", Proc of 32nd Int. Cosmic Ray Conf., Beijing, China 2 (2011) 55, arXiv:1107.4805 [astro-ph.HE]. 
[8] Pierre Auger Collaboration, J. Abraham et al., "Upper limit on the diffuse flux of UHE tau neutrinos from the Pierre Auger Observatory", Phys. Rev. Lett. 100 (2008) 211101, arXiv:0712.1909 [astro-ph].

[9] Pierre Auger Collaboration, P. Abreu et al., "A Search for Ultra-High Energy Neutrinos in Highly Inclined Events at the Pierre Auger Observatory", Phys. Rev. D84 (2011) 122005, arXiv:1202.1493 [astro-ph.HE].

[10] Pierre Auger Collaboration, P. Abreu et al.,"Search for point-like sources of ultra-high energy neutrinos at the Pierre Auger Observatory and improved limit on the diffuse flux of tau neutrinos", Astrophys.J. 755 (2012) L4, arXiv:1210.3143 [astro-ph.HE].

[11] Pierre Auger Collaboration, P. Abreu et al., "Ultrahigh Energy Neutrinos at the Pierre Auger Observatory", Adv.High Energy Phys. 2013 (2013) 708680, arXiv:1304.1630 [astro-ph.HE].

[12] Pierre Auger Collaboration, P. Abreu et al., "Searches for anisotropies in the Arrival directions of the highest energy cosmic rays", ApJ 804, 15 (2015)

[13] Pierre Auger Collaboration, P. Abreu et al., "Depth of maximum of air-showers profiles at the Auger Observatory: measurements at energies above $10^{17.8} \mathrm{eV}^{\prime \prime}$, Physical Review D 90, 122005 (2014)

[14] C. Tsallis "Possible generalization of Boltzmann-Gibbs entropy" J. Stat. Phys. 52,479 (1988).

[15] B. I. Abelev et al. (STAR Collaboration), "Strange particle production in $p+p$ collisions at $\sqrt{s}=200$ GeV", Phys. Rev. C75, 064901 (2007).

[16] A. Adare et al. (PHENIX Collaboration), "Identified charged hadron production in $p+p$ collisions at $\sqrt{s}=200$ and 62.4 GeV", Phys. Rev. C83, 052004, (2010); Phys. Rev. C 83, 064903 (2011).

[17] K. Aamodt, et al. (ALICE Collaboration), "Production of pions, kaons and protons in pp collisions at $\sqrt{s}=900 \mathrm{GeV}$ with ALICE at the LHC", Eur. Phys. J. C71 (2011) 1655 .

[18] G. Aad, et al. (ATLAS Collaboration), "Charged-particle multiplicities in pp interactions measured with the ATLAS detector at the LHC", New J. Phys. 13 (2011) 053033.

[19] V. Khachatryan, et al. (CMS Collaboration), "Strange Particle Production in pp Collisions at $\sqrt{s}=0.9$ and $7 \mathrm{TeV}$ ", JHEP 1105 (2011) 064 .

[20] J. Cleymans, G. I. Lykasov, A. S. Parvan, A. S. Sorin, O. V. Teryaev and D. Worku,"Systematic properties of the Tsallis Distribution: Energy Dependence of Parameters in High-Energy p-p Collisions.", Phys. Lett. B723, 351 (2013) [arXiv:1302.1970 [hep-ph]].

[21] J. Cleymans, J. Phys. Conf. Ser. 455, 012049 (2013); M. D. Azmi and J. Cleymans, arXiv:1311.2909 [hep-ph]; M. D. Azmi and J. Cleymans, arXiv:1401.4835 [hep-ph].

[22] C. Y. Wong and G. Wilk, "Tsallis Fits to $p_{T}$ Spectra and Multiple Hard Scattering in pp Collisions at the LHC", Phys. Rev. D87, 114007 (2013) [arXiv:1305.2627 [hep-ph]].

[23] C. Y. Wong and G. Wilk, "Tsallis Fits to $p_{T}$ Spectra for pp Collisions at LHC", Acta Phys. Polon. B42, 2047 (2012) [arXiv:1210.3661 [hep-ph]].

[24] G. Wilk and Z. Wlodarczyk, "Interpretation of the Nonextensivity Parameter q in Some Applications of Tsallis Statistics and Lévy Distributions", Phys. Rev. Lett. 84, 2770 (2000) [hep-ph/9908459].

[25] L. Marques, J. Cleymans, A. Deppman, "Description of High-Energy pp Collisions Using Tsallis Thermodynamics: Transverse Momentum and Rapidity Distributions", Phys.Rev. D91 (2015) 054025 [arXiv:1501.00953 [hep-ph]]. 
[26] Bao-Chun Li, Ya-Zhou Wang, Fu-Hu Liu, Xin-Jian Wen, You-Er Dong, "Particle production in relativistic $p p(\bar{p})$ and AA collisions at RHIC and LHC energies with Tsallis statistics using the two-cylindrical multisource thermal model", Phys.Rev. D89 (2014) 5, 054014 [arXiv:1403.4025 [hep-ph]].

[27] Bao-Chun Li, Zhao Zhang, Jun-Hui Kang, Guo-Xing Zhang, Fu-Hu Liu, "Tsallis Statistical Interpretation of Transverse Momentum Spectra in High-Energy pA Collisions", Adv.High Energy Phys. 2015 (2015) 741816.

[28] G. Wilk and Z. Wlodarczyk, "Power laws in elementary and heavy-ion collisions: A Story of fluctuations and nonextensivity?", Eur.Phys.J. A40 (2009) 299-312.

[29] D. Heck, G. Schatz, T. Thouw, J. Knapp and J. N. Capdevielle, Report FZKA 6019 (1998). Forschungszentrum Karlsruhe; http://wwwik.fzk.de/corsika/physics description/corsika phys.html.

[30] E. J. Ahn, R. Engel, T. K. Gaisser, P. Lipari and T. Stanev, Phys. Rev. D 80, 094003 (2009).

[31] H. Fesefeldt, Report PITHA-85/02 (1985), RWTH Aachen.

[32] Hagedorn, R., Suppl. Nuovo Cimento 3, 147-186 (1965) 